\title{
A Review of Exenatide: Optimizing Glycemic Control and Associated Cardiovascular Risk Factors in Type 2 Diabetes
}

Zin Z. Htike $\cdot$ Kamlesh Khunti $\cdot$ Melanie Davies

To view enhanced content go to www.diabetestherapy-open.com

Received: December 2, 2011 / Published online: March 16, 2012

(C) The Author(s) 2012. This article is published with open access at Springerlink.com

\section{ABSTRACT}

Type 2 diabetes mellitus (T2DM) is a wellrecognized risk factor for the development of cardiovascular disease. With an increasing prevalence of obesity, this risk has increased further. Management of T2DM in obese patients is particularly challenging as treatment with the majority of glucose-lowering agents results in weight gain. Thus, the development of a

\section{Z. Z. Htike}

Department of Diabetes and Endocrinology, Leicester Royal Infirmary, University Hospitals of Leicester NHS Trust, Leicester, UK

\section{K. Khunti}

Department of Health Sciences,

University of Leicester, Leicester, UK

M. Davies ( $\square)$

Department of Cardiovascular Sciences, University of Leicester, Leicester Royal Infirmary, c/o Victoria Building, Leicester LE1 5WW, UK

e-mail: Melanie.davies@uhl-tr.nhs.uk

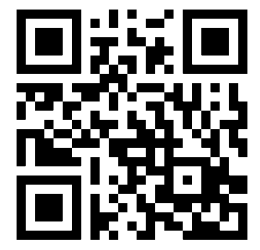

Enhanced content for this article is available on the journal web site: www.diabetestherapy-open.com therapeutic option which could improve glycemic control without weight gain or hypoglycemia, such as the glucagon-like peptide-1 (GLP-1) analog exenatide, is a welcome addition to the currently available therapies in the management of T2DM. With recognition and better understanding of the role of incretin hormones in T2DM, exenatide was developed and introduced into clinical practice in 2005. Both randomized controlled trials and retrospective observational studies have shown that treatment with exenatide not only improves glycemic control, with a low risk of hypoglycemia, but also results in concurrent weight loss and the additional benefit of improvement in cardiovascular risk factors. This article will provide an overview of both short- and long-acting exenatide in the management of T2DM and associated cardiovascular risk factors.

Keywords: Cardiovascular risk factors;

Diabetes; Exenatide; Extended-release;

GLP-1 analog; Glycemic control;

Hypoglycemia; Incretin mimetics;

Type 2 diabetes - Obesity 


\section{INTRODUCTION}

Diabetes is a chronic disorder affecting 285 million adults worldwide, a figure that is predicted to rise to an estimated 439 million over the next 20 years [1]. In the UK alone, 2.6 million people are affected, of whom approximately $90 \%$ have type 2 diabetes mellitus (T2DM).

T2DM is characterized by beta-cell dysfunction and a varying degree of insulin resistance. Obesity per se is the major contributory factor in development of insulin resistance and impaired glucose tolerance. Both obesity and poor glycemic control in T2DM, either in combination or independent of each other, increase the risk of cardiovascular disease and mortality [2].

An integral part of the management strategy in T2DM is lifestyle modification. Medical therapy with oral glucose-lowering agents is then instituted in a stepwise approach, followed by initiation and then intensification of insulin therapy $[3,4]$. Because the efficacy of most existing glucose-lowering agents reduces over time and beta-cell function continues to decline, glycemic targets are often not achieved. Moreover, the use of these agents is often limited by undesirable side effects, such as weight gain, hypoglycemia, fluid retention, and gastrointestinal (GI) intolerance. This subsequently leads to poor adherence to medication on the part of the patient, or reluctance to optimize medication on the part of the clinician, and hence to many patients not achieving glycemic targets in routine clinical practice.

The development of novel agents, such as incretin-based therapies, that provide improved glycemic control without weight gain and with a low risk of hypoglycemia are a welcome addition to the clinician's armamentarium.
A literature search was carried out using Medline and PubMed from March 1, 2011 to December 22, 2011. This review provides an overview of the role of exenatide, one of the incretin-based agents, in the management of T2DM and its effect on associated cardiovascular risk factors as seen in both clinical trials and real-life observational studies.

\section{INCRETIN-BASED THERAPIES}

Incretins are gut hormones produced by the small intestine in response to oral ingestion of glucose, which accounts for more than $50 \%$ of insulin secretion after food intake. These hormones include glucose-dependent insulinotropic polypeptide (GIP) and glucagon-like peptide-1 (GLP-1). The higher insulin response to orally administered glucose than to parenterally administered glucose is known as the incretin effect, which is deemed to be impaired in T2DM [5]. Physiologically, GLP-1 is rapidly inactivated by the enzyme dipeptidyl peptidase-4 (DPP-4) and, hence, its action is short-lived. Thus, targeting GLP-1, either to increase its availability or to prolong its action, has become an attractive option in developing new therapeutic agents in the management of T2DM.

Currently, two classes of incretin-based therapy are available, the incretin enhancers and the incretin mimetics. The incretin enhancers are DPP-4 inhibitors (sitagliptin, saxagliptin, vildagliptin, linagliptin, allogliptin) that prevent the degradation of GLP-1 by inhibiting the DPP-4 enzyme, whilst the incretin mimetics are GLP-1 analogs (exenatide and liraglutide) that directly act on GLP-1 receptors (GLP-1R).

GLP-1 promotes the glucoregulatory effect by increasing insulin secretion and suppressing 
glucagon. It also delays gastric emptying and acts centrally on the satiety center to reduce the food intake, thus facilitating weight loss [5]. Some studies have shown it to increase beta-cell proliferation and reduce apoptosis [5]. This may have an influence in delaying the disease progression.

There is emerging evidence suggesting that GLP-1 has a direct influence on myocardium and vascular endothelium, which could potentially improve cardiovascular outcomes, either via GLP-1R or independently of GLP-1R. The exact mechanism underlying the cardioprotective effect has not been fully elucidated. Some studies suggest an increased myocardial sensitivity to insulin and myocardial glucose uptake independently of plasma insulin level [6]. Other studies indicate an inhibition of cardiac myocyte apoptosis via inflammatory mediators and activation of antioxidant genes to reduce fibrosis and left ventricular remodeling, thus restoring left ventricular function after myocardial infarction [7]. A review describes improvement in left ventricular function and myocardial oxygen consumption with GLP-1 infusion in chronic heart failure, myocardial infarction, coronary angioplasty, and coronary artery bypass graft, although study patient populations were small in number [8].

GLP-1 produces a vasodilatory effect through nitric oxide (NO) production in the endothelium, a mechanism independent of GLP-1R, in an isolated mouse heart model [9]. However, this vasodilatory effect is also observed regardless of the NO pathway, indicating a direct action of GLP-1 on vascular smooth muscle through the GLP-1R [10]. Furthermore, administration of exenatide was found to be associated with a reduction of inflammatory mediators, such as the highsensitivity C-reactive protein [11].

\section{GLP-1 ANALOGS}

Currently, both the US Food and Drug Administration (FDA) and the European Medications Agency (EMA) have approved GLP-1 analogs to be used in the management of T2DM. Exenatide (Byetta ${ }^{\circledR}$ ) was the first synthetic GLP-1 analog, approved by the FDA in 2005 and recommended by the National Institute for Health and Clinical Excellence (NICE) in 2008 [3]. The parent compound was derived from exendin, found in the saliva of the desert Gila monster (Heloderma suspectum). It shares approximately 50\% homology with human GLP-1 and resists inactivation by the DPP-4 enzyme, resulting in a longer half-life $(2.4 \mathrm{~h})$ and duration of action (4-6 h). It is usually administered subcutaneously at a dose of $5 \mu \mathrm{g}$ twice daily (BID) and titrated up to $10 \mu \mathrm{g}$ (BID) after an initial 4 weeks.

The NICE guidance advocates the use of exenatide twice daily (ExBID) as an adjunctive therapy to oral hypoglycemic agents in triple therapy with metformin and either a sulfonylurea (SU) or a thiazolidinedione (TZD) in specified patients with T2DM whose glycemic control remains inadequate [3].

The second GLP-1 analog, liraglutide $\left(\right.$ Victoza $\left.^{\circledR}\right), \quad$ is a once-daily formulation, synthesized by recombinant DNA technology, sharing 97\% homology with human GLP-1. It was recommended by the NICE in the technology appraisal guidance in 2010 [12].

Recently the EMA has approved the third GLP-1 analog, extended-release exenatide (ExER) (Bydureon ${ }^{\circledR}$ ), $2 \mathrm{mg}$, as a once-weekly preparation [13]. The active medication is encapsulated into polymer-based microspheres that are injected into the body where they degrade slowly, gradually releasing the drug to provide continuous therapeutic exenatide levels. Thus, there is less peak-trough 
variation; however, injection site reactions, pruritus, and nodules are more common than with ExBID.

The use of ExER has not been updated in the NICE guidance as of yet. However, the evidence for both short- and long-acting exenatide on glycemic control and weight reduction, and the cardiovascular effects, will be discussed in this review article.

\section{Metabolic Effect of Exenatide on Glycemic Control and Body Weight}

\section{Efficacy of ExBID}

Efficacy as an Add-on Therapy to Oral Antidiabetic Agents The safety and efficacy of adding ExBID to a single oral antidiabetic agent (OAD) or combination of commonly used OADs were investigated in the three AC2993: Diabetes Management for Improving Glucose Outcomes (AMIGO) studies [14-16]. The studies were similar in core design in subjects with T2DM with inadequate glycemic control on a maximum tolerated dose of either metformin or a SU alone, or a combination of the two. The studies were triple-blind, placebo-controlled trials with three study arms: placebo, ExBID $(5 \mu \mathrm{g})$, and ExBID $(10 \mu \mathrm{g})$.

In all the studies, addition of ExBID $(10 \mu \mathrm{g})$ resulted in a significant improvement in glycemic control of approximately $1 \%$ and a dose-dependent progressive weight loss of approximately -1.5 to $-3.0 \mathrm{~kg}$. There was a statistically significant decrease in mean fasting plasma glucose of $-0.6 \mathrm{mmol} / \mathrm{L}$. The postprandial plasma glucose level was significantly reduced at week 4 and this was sustained up to 30 weeks in the standardized meal tolerance test [14-16].

Furthermore, 32\% (314) of the participants in the above studies completed the 82-week open-label extension. A reduction in glycated hemoglobin $\left(\mathrm{HbA}_{1 \mathrm{c}}\right)$ at 30 weeks was found to be sustained at 82 weeks (1.1\%) with a progressive weight reduction of $-4.4 \mathrm{~kg}$ [17]. In the open-label follow-up, a subset of patients $(n=217)$ achieved 3.5 years of ExBID exposure and maintained a significant $\mathrm{HbA}_{1 \mathrm{c}}$ reduction of $1 \%(P<0.0001)$ and weight loss of $-5.3 \mathrm{~kg}$ $(P<0.0001)$ [18].

When ExBID was added to a TZD, the combination was also found to be effective. TZDs are insulin sensitizers, which improve insulin action in muscle and adipose tissue with some reduction in hepatic glucose production. These complementary mechanisms rationalize the potential use of combining the two in management of T2DM. In a double-blind, placebo-controlled trial, patients with T2DM who were suboptimally controlled with TZD (with or without metformin) were investigated over a period of 16 weeks [19]. Addition of ExBID resulted in a placebo-subtracted reduction in $\mathrm{HbA}_{1 \mathrm{c}}$ of $0.98 \%$ and a significant weight loss of $-1.51 \mathrm{~kg}(P<0.001)$. However, this study was of relatively short duration. Liutkuz et al. [20] reported a similar improvement in glycemic control in a study over a 26 -week period.

When the efficacy of ExBID was compared with TZDs in a meta-analysis including 22 studies over a 24 -week period, TZDs were found to produce a greater improvement in glycemic control (reduction in $\mathrm{HbA}_{1 \mathrm{c}}$ of -0.80 vs. $-0.60 \%)$, whilst exenatide was associated with a reduction in body weight $(-2.74$ vs. $+2.19 \mathrm{~kg}$ ). A significant reduction in fasting plasma glucose was observed only in those treated with TZDs, with a mean difference of $-1.64 \mathrm{mmol} / \mathrm{L}$ [21].

Efficacy as an Alternative Therapy to Insulin The NICE has endorsed the use of exenatide as a third-line agent and an 
alternative to insulin in the management of T2DM, particularly in obese subjects [3]. The efficacy of ExBID compared to basal insulin glargine or biphasic insulin aspart is summarized in Table 1 [14-16, 19, 20, 22-27].

In a head-to head, open-label study, 551 patients with T2DM who were not adequately controlled on metformin or a SU were randomized to receive treatment with either ExBID or basal insulin glargine over 26 weeks. Both treatments produced a comparable reduction in $\mathrm{HbA}_{1 \mathrm{c}}$ of $1.11 \%$. However, there was a significant difference in body weight of $-4.1 \mathrm{~kg}$ between the two groups, a decrease of $-2.3 \mathrm{~kg}$ with ExBID and an increase of $+1.8 \mathrm{~kg}$ with glargine [22]. A similar efficacy in glycemic control with divergent effects on body weight in favor of ExBID were also reported in another multicenter, randomized, open-label, parallelcomparator study (Helping Evaluate Exenatide in patients with diabetes compared with LongActing insulin [HEELA]) (Table 1) [23]. A smaller study with a longer duration of 52 weeks also reported similar results [24].

Barnett and colleagues conducted a crossover study where 138 patients were randomized to receive either ExBID followed by insulin glargine or vice versa over successive 16-week periods. Glycemic control improved in both groups by 1.36\%; however, patients lost weight while on ExBID and gained weight on insulin glargine, with a difference of $-2.2 \mathrm{~kg}$ [25]. In all three studies, ExBID reduced prandial glucose excursions more than glargine, while glargine reduced fasting glucose concentrations more than ExBID [23-25]. When compared to treatment with biphasic insulin aspart, both treatments showed similar improvements in $\mathrm{HbA}_{1 \mathrm{c}}$ of approximately $1 \%$, but showed a weight difference of $-5.4 \mathrm{~kg}$ in favor of ExBID [26].

Thus, the evidence to date has demonstrated that treatment with ExBID was noninferior to that of basal insulin or biphasic insulin in optimizing the glycemic control. In contrast to the weight gain observed with insulin, ExBID therapy has an added benefit of weight loss.

Efficacy as an Add-on Therapy to Insulin At present, the combined use of insulin and a GLP-1 analog is not licensed. In a nationwide audit in the UK, of the 4,857 patients treated with ExBID, nearly $40 \%(1,921)$ were on combination with insulin [28]. However, there is limited randomized data on the combined use of these therapies.

Buse and colleagues studied the efficacy of adding ExBID to basal insulin glargine in a randomized, placebo-controlled trial. Patients with T2DM whose glycemic control was not adequate on insulin glargine alone or in combination with metformin or pioglitazone were randomized into groups receiving either $\operatorname{ExBID}(n=138)$ or placebo $(n=123)$. Addition of ExBID significantly reduced $\mathrm{HbA}_{1 \mathrm{c}}$ $(-1.74$ vs. $-0.93 \%, P<0.001)$ without increased hypoglycemia. Mean body weight also decreased by $-1.8 \mathrm{~kg}$ with exenatide and increased by $+1 \mathrm{~kg}$ with placebo [27].

In a retrospective, real-life clinical study of 188 patients with T2DM who were receiving a combination of insulin and ExBID over 27 months, the greatest reduction in $\mathrm{HbA}_{1 \mathrm{c}}$ was observed in the first 6 months $(-0.66 \%)$ and the reduction was maintained at $-0.54 \%$ over the next 18 months [29]. Mean body weight also progressively declined to $-6.2 \mathrm{~kg}$ up to 18 months. A 1-year follow-up of 124 subjects from another observational study showed a significant $-0.87 \% \quad(P<0.001)$ reduction in $\mathrm{HbA}_{1 \mathrm{c}}$ and $-5.2 \mathrm{~kg} \quad(P<0.001)$ reduction in body weight when the combination was used [30].

By study design, in the randomized, controlled trial, basal insulin requirement 


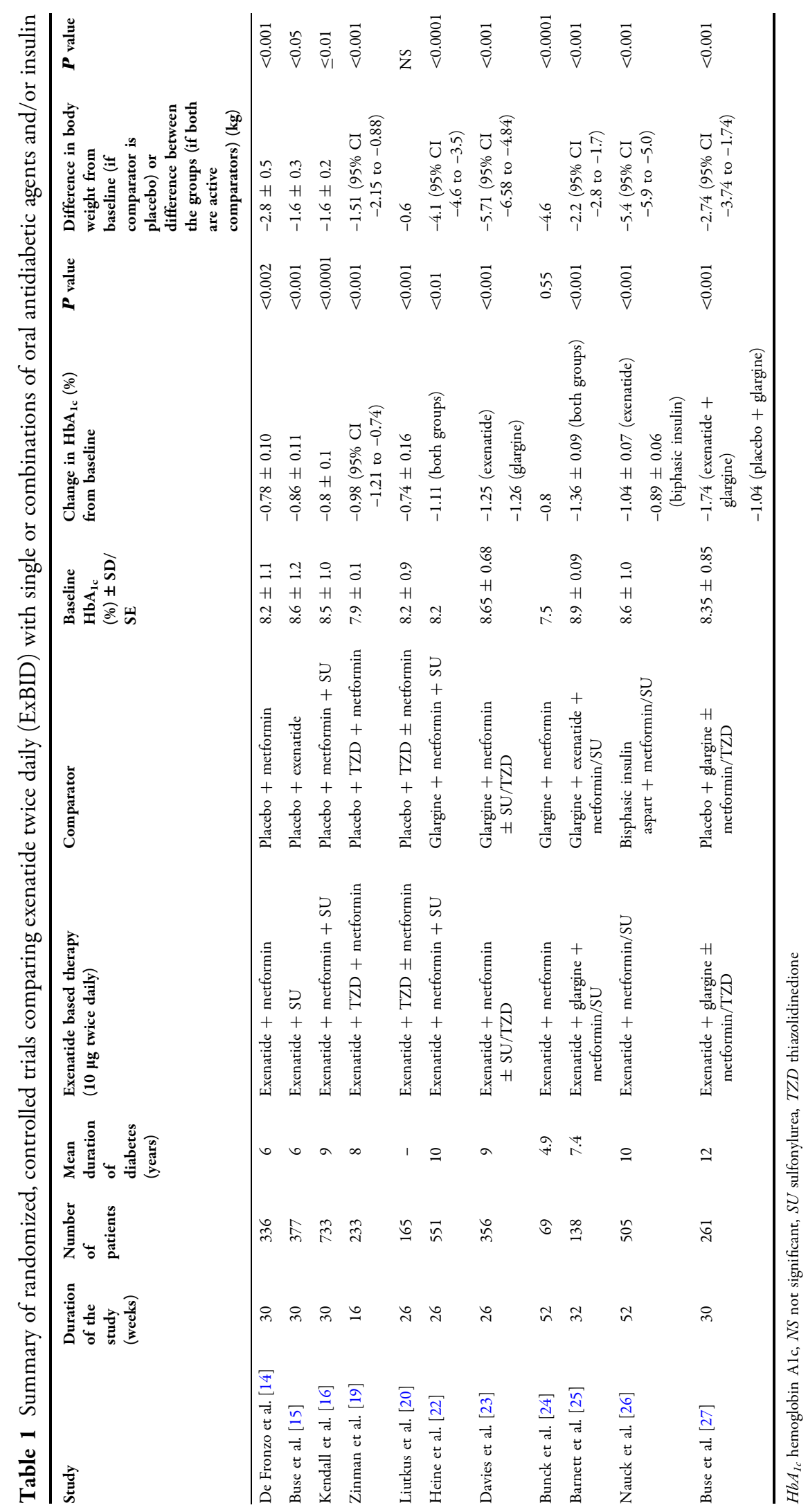


increased from baseline in both groups taking exenatide or placebo, but the increase was greater in the placebo group ( +20 vs. $+13 \mathrm{U} /$ day) [27]. However, in the real-life study, the mean total daily dose of insulin was decreased in all patients up to 12 months (-14.8 U/day). The changes thereafter were not statistically significant. The mean prandial insulin dose also decreased from baseline, ranging from -29.7 to $-55.7 \%$ over 27 months, but with no significant change in basal insulin dose [29]. A greater and significant reduction in total daily dose of $45 \mathrm{U} /$ day $(P<0.001)$ was reported in the nationwide UK audit [28].

In conclusion, the addition of exenatide to an insulin-based therapy in obese patients with inadequately controlled T2DM is associated with a reduction in mean $\mathrm{HbA}_{1 \mathrm{c}}$ and body weight in both randomized and real-life studies. The combination of basal insulin and exenatide appears to be a logical approach, as the former improves fasting glucose level while the latter deals with postprandial glucose excursion. However, the combined use is not licensed at present.

Efficacy as an Alternative to Liraglutide The rationale of developing a longer-acting preparation of GLP-1 is discussed above ("GLP1 Analogs"). When ExBID was directly compared against the longer-acting GLP-1 analog, liraglutide once daily, liraglutide showed greater glycemic efficacy with a greater reduction in $\mathrm{HbA}_{1 \mathrm{c}}(-1.12$ vs. $-0.79 \%$, $P<0.0001)$ and fasting glucose level $(-1.16$ vs. $-0.60 \mathrm{mmol} / \mathrm{L}$ ). More patients also achieved $\mathrm{HbA}_{1 \mathrm{c}} \leq 7 \%$ with liraglutide (54 vs. 43\%, $P=0.0015)$. Both drugs promoted a similar weight loss of approximately $3 \mathrm{~kg}$ over 26 weeks. However, minor hypoglycemic episodes were less frequent and GI intolerance was lower with liraglutide (Liraglutide Once
Daily Compared with Exenatide Twice Daily [LEAD 6] Study) [31]. However, the liraglutide dose used $(1.8 \mathrm{mg})$ is higher than the dose recommended by the NICE $(1.2 \mathrm{mg})$, and to date there is no head-to-head study comparing ExBID with liraglutide $1.2 \mathrm{mg}$.

\section{Efficacy of ExER}

Although GLP-1 is mainly secreted postprandially, there is evidence suggesting that GLP-1 may play a role in the control of basal insulin and, hence, fasting glucose concentration. In a small, randomized, placebo-controlled trial, continuous infusion of GLP-1 over $24 \mathrm{~h}$ in T2DM produced a significant reduction in both nocturnal and fasting glucose levels compared to a 16-h infusion [32]. This demonstrates the impact of continuous GLP-1 exposure on overall glycemic control by reducing both fasting and prandial glucose. This rationale also led to further exploration and development of extendedreleased preparations.

Furthermore, unlike quick-acting exenatide, a reduced peak concentration with a slow-release preparation could potentially reduce the GI intolerance. In addition to increased efficacy and tolerability, a long-acting preparation offers the convenience of once-weekly administration, which could enhance patient adherence. The findings from phase 3 clinical trials assessing the efficacy of ExER ( $2 \mathrm{mg}$ once a week) against ExBID, OADs, or basal insulin glargine are summarized in Table 2 [33-38].

Efficacy of ExER Compared to ExBID The safety and efficacy of ExER compared to ExBID were compared in the Diabetes therapy Utilization: Researching changes in $\mathrm{A}_{1 \mathrm{C}}$, weight, and other factors Through Intervention with exenatide once weekly (DURATION) 1 study [33]. The reduction in $\mathrm{HbA}_{1 \mathrm{c}}$ 
( -1.9 vs. $-1.5 \%, P=0.0023)$ and fasting glucose ( -2.3 vs. $-1.4 \mathrm{mmol} / \mathrm{L}, P<0.0001)$ were greater with ExER than with ExBID. However, the reduction in postprandial glucose was less pronounced with ExER. A progressive and significant weight reduction occurred in both treatment groups but with no difference between groups $(-0.1 \mathrm{~kg}, \quad P=0.89)$. Similar findings were reported in the DURATION 5 study (Table 2) [37].

On completion of the initial 30-week DURATION 1 study, it was extended for a further 22-week period. Patients receiving ExER continued the same treatment (ExER group), whereas those on ExBID were switched to ExER (ExBID $\rightarrow$ ExER group). Patients continuing ExER maintained a $\mathrm{HbA}_{1 \mathrm{c}}$ reduction and those switched from ExBID to ExER achieved a further improvement in $\mathrm{HbA}_{1 \mathrm{c}}$ of $0.2 \%$. Both groups displayed the same reduction with a mean $\mathrm{HbA}_{1 \mathrm{c}}$ of $6.6 \%$ at the end of the study period. Weight loss was -4.1 and $-4.5 \mathrm{~kg}$, respectively, in the ExER group and ExBID $\rightarrow$ ExER group [39].

In both studies, a greater proportion of patients receiving ExER achieved the target $\mathrm{HbA}_{1 \mathrm{c}}$ of $\leq 7 \%$. A greater reduction in $\mathrm{HbA}_{1 \mathrm{c}}$ with ExER illustrates the effect of continuous exposure to exenatide over $24 \mathrm{~h}$ compared to short exposure with ExBID. In conclusion, both studies demonstrate the difference in glycemic efficacy of long-acting compared to short-acting exenatide, and treatment with ExER proved to be superior to that with ExBID.

Efficacy of ExER Compared to a DPP-4 Inhibitor, TZD, and Metformin The DURATION 2 study investigated the safety and efficacy of ExER against the maximum approved dose of a DPP-4 inhibitor or TZD in patients treated with metformin. In this double-blind, superiority trial, patients were randomized into either an ExER (2 mg), a sitagliptin (100 mg), or a pioglitazone $(45 \mathrm{mg})$ arm. Treatment with ExER reduced $\mathrm{HbA}_{1 \mathrm{c}}(-1.5 \%)$ more significantly than sitagliptin $(-0.9 \%)$ or pioglitazone $(-1.2 \%)$. The reduction in fasting glucose was significantly greater with ExER $(-1.8 \mathrm{mmol} / \mathrm{L})$ than with sitagliptin $(-0.9 \mathrm{mmol} / \mathrm{L})$, but not with pioglitazone $(-1.5 \mathrm{mmol} / \mathrm{L})$. Similarly, a greater reduction in body weight was observed with ExER, a treatment difference of $-1.5 \mathrm{~kg}$ with sitagliptin $(P=0.0002)$ and $-5.1 \mathrm{~kg}$ with pioglitazone $(P<0.0001)$ [34]. Thus, the addition of ExER to metformin achieved better glycemic control and weight loss than sitagliptin or pioglitazone.

The DURATION 4 study reported the efficacy of ExER as a monotherapy compared to the maximum tolerated dose of sitagliptin, pioglitazone, or metformin monotherapy in drug-naïve T2DM patients [36]. The reduction in $\mathrm{HbA}_{1 \mathrm{c}}$ was found to be greater with pioglitazone monotherapy $(-1.63 \%)$ and less with sitagliptin $(-1.15 \%)$. Both metformin and ExER produced a similar reduction in $\mathrm{HbA}_{1 \mathrm{c}}$ of approximately $-1.5 \%$. Thus, in contrast to combination therapy with metformin in the DURATION 2 study, ExER monotherapy was not superior to that of pioglitazone in this study. A decrease in weight of approximately $-2 \mathrm{~kg}$ was reported with both ExER and metformin, and $-0.8 \mathrm{~kg}$ with sitagliptin, whilst an increase of $+1.5 \mathrm{~kg}$ was reported with pioglitazone. It was concluded that treatment with ExER provides an option for the initial treatment of T2DM in drug-naïve patients. However, ExER is not licensed for monotherapy.

\section{Efficacy of ExER Compared to Insulin} Glargine The efficacy of ExER was compared with insulin glargine in the open-label, randomized (DURATION 3) study. T2DM patients with inadequate control who were taking metformin or the combination of 


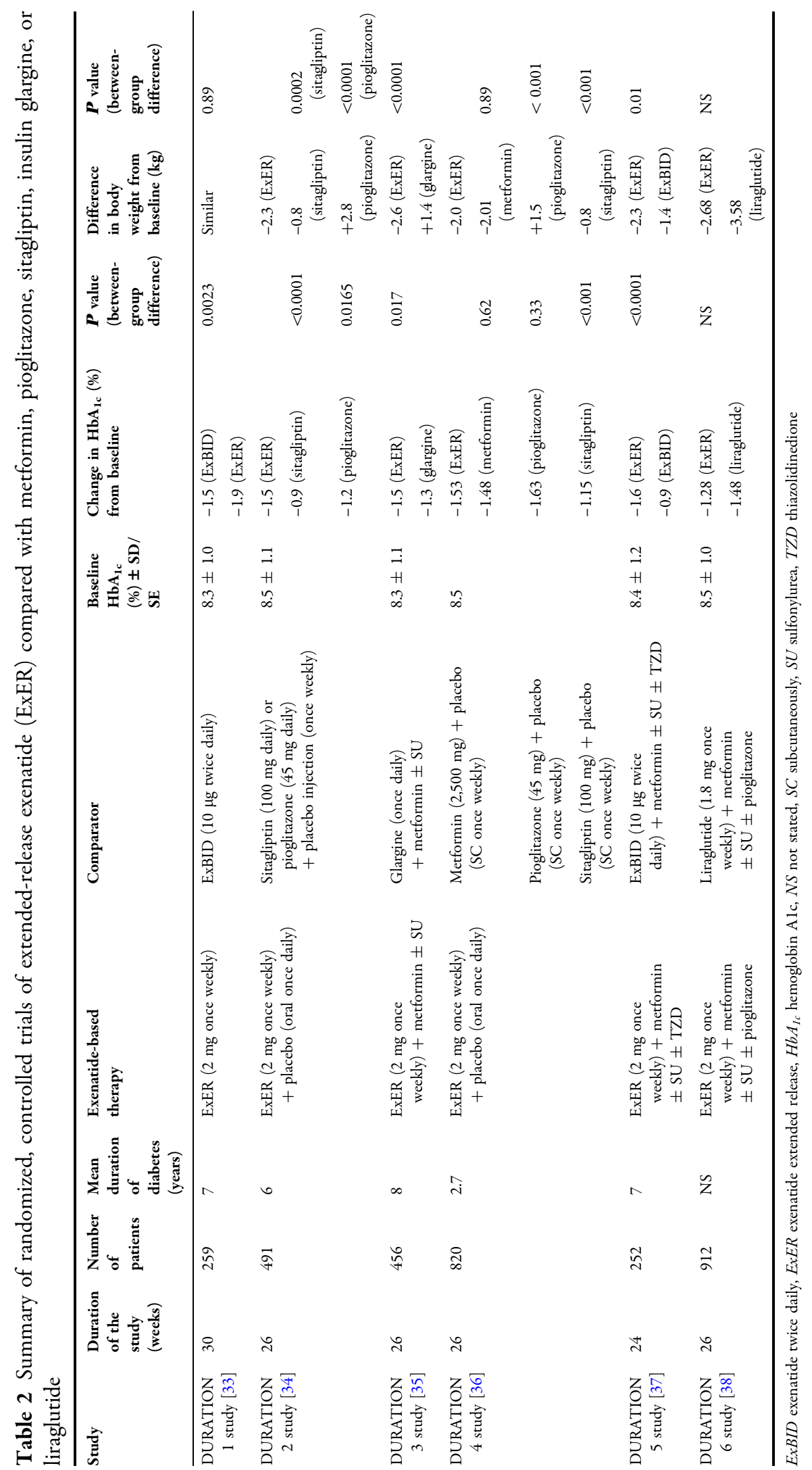


metformin and a SU were randomized to receive either ExER $(n=233)$ or glargine $(n=223)$ over 26 weeks. Treatment with ExER was found to be associated with a greater reduction in $\mathrm{HbA}_{1 \mathrm{c}}$ ( -1.5 vs. $-1.3 \%, P=0.017)$ and progressive weight loss than with glargine, a treatment difference of $-4 \mathrm{~kg}(P<0.0001)$ [35]. Insulin glargine produced a greater reduction in fasting glucose $(-2.8$ vs. $-2.1 \mathrm{mmol} / \mathrm{L}, \quad P=0.001)$, while a significantly greater reduction in postprandial glucose was obtained with ExER. The mean insulin dose of glargine increased from $10 \mathrm{U} /$ day at baseline to $31 \mathrm{U} /$ day at 26 weeks. At the endpoint, a greater proportion of patients receiving ExER achieved $\mathrm{HbA}_{1 \mathrm{c}}<7 \%$ (60 vs. 48\%). Thus, treatment with ExER was superior to that with insulin glargine.

\section{Efficacy of ExER Compared to Liraglutide A} randomized, head-to-head trial comparing ExER $(n=461)$ with liraglutide $1.8 \mathrm{mg}(n=451)$ was undertaken in the DURATION 6 study. This 26-week, open-label, noninferiority study enrolled patients with T2DM who were not achieving adequate $\mathrm{HbA}_{1 \mathrm{c}}$ control with combined OADs. Treatment with liraglutide resulted in a greater reduction in $\mathrm{HbA}_{1 \mathrm{c}}(-1.48$ vs. $-1.28 \%)$ and body weight ( -3.5 vs. $-2.68 \mathrm{~kg}$ ) compared to ExER. More subjects taking liraglutide achieved $\mathrm{HbA}_{1 \mathrm{c}}<7 \% \quad(60.2$ vs. 52.3\%, $P=0.008$ ) [38]. Thus, ExER did not meet the prespecified primary endpoint of noninferiority to liraglutide.

In summary, treatment with ExER has demonstrated a consistent improvement in both $\mathrm{HbA}_{1 \mathrm{c}}$ and body weight. Thus, ExER is a promising long-acting GLP-1 analog with an efficacy superior to that of ExBID, insulin glargine, or the maximum approved dose of sitagliptin and pioglitazone. However, compared to the once daily GLP-1 analog liraglutide at a dose of $1.8 \mathrm{mg}$, there was a lesser reduction in $\mathrm{HbA}_{1 \mathrm{c}}$ and better GI tolerance.

\section{Metabolic Effect of Exenatide on Cardiovascular Parameters}

It is well established that T2DM is a significant risk factor for cardiovascular disease (CVD), and improvement in glycemic control reduces both microvascular and macrovascular complications. The United Kingdom Prospective Diabetes Study (UKPDS) showed a 16\% risk reduction for myocardial infarction which remained significant in a 10 -year post-study follow-up of patients with intensive glycemic control $(P=0.01)$ [40]. The finding of increased higher mortality in the intensive glycemic control group of the Action to Control Cardiovascular Risk in Diabetes (ACCORD) trial was controversial, although nonfatal myocardial infarction occurred less in this group [41]. However, the Action in Diabetes and Vascular Disease (ADVANCE) trial showed a small but significant reduction in the incidence of both microvascular and macrovascular complications with intensive glucose lowering (hazard ratio $[\mathrm{HR}]=0.09, P=0.01$ ) [42]. Furthermore, postprandial hyperglycemia is strongly associated with CVD and has been regarded by some as a more important CVD risk factor than fasting hyperglycemia [43].

In patients with diabetes, intentional weight loss is associated with a $28 \%$ reduction in CVD and diabetes-related mortality [44]. Thus, weight loss rendered by treatment with either ExBID or ExER could potentially offer the beneficial effect of reducing CVD events and related mortality independent of other mechanisms mentioned previously. In a large, retrospective analysis performed on the Lifelink database, patients treated with ExBID, despite higher rates of coronary artery disease, obesity, 
hypertension, and hyperlipidemia, were less likely to have a CVD event $(\mathrm{HR}=0.81, P=0.01)$ and had lower rates of CVD-related hospitalization (HR $=0.88, P=0.02)$ than patients not treated with exenatide [45].

Peskin and associates studied the long-term effects of ExER by employing a validated mathematical Archimedes Model on data derived from the 52-week clinical trial. Treatment with simulated ExER resulted in an earlier benefit and two- to three-times greater relative reductions in major CVD events by improving glycemic control and weight (EQw model-1), and by improving blood pressure and lipids (EQw model-2) with relative risk reductions of 12 and 17\%, respectively, over 20 years [46]. The EXenatide Study of Cardiovascular Event Lowering (EXSCEL) is currently recruiting and is expected to complete by 2016 [47]. It is designed to determine whether treatment with ExER is associated with improved cardiovascular outcomes compared to standard care with traditional diabetes medication.

\section{Effects of Exenatide on Blood Pressure}

The mechanism for lowering blood pressure by exenatide appears to be related to weight loss. However, in salt-sensitive rodent models, GLP-1 treatment has been shown to have antihypertensive, cardioprotective, and renoprotective actions through natriuretic and diuretic effects [48]. As discussed previously, the action on endothelium and vascular smooth muscle, producing vasodilatory effects, may contribute to lowering of the blood pressure $[9,10]$.

In the follow-up, open-label extension of the three AMIGO studies, addition of ExBID resulted in an improvement of both systolic blood pressure (SBP) and diastolic blood pressure (DBP), by -3.5 and $-3.3 \mathrm{mmHg}$, respectively [18]. In a pooled analysis of six trials involving 2,171 subjects, treatment with ExBID over 6 months showed greater reductions in SBP, mainly in patients with high baseline SBP, compared to placebo $(-2.8 \mathrm{mmHg}, P=0.0002)$ or insulin $(-3.7 \mathrm{mmHg}, P<0.0001)$, with no effect on DBP [49].

In the HEELA study, there was a significant decrease in SBP with ExBID treatment, which was statistically and significantly different from the change with insulin glargine $(-3.6 \mathrm{mmHg}$, $P=0.034)$ [23]. Both SBP $(-2.7 \mathrm{mmHg})$ and DBP $(-1.7 \mathrm{mmHg})$ decreased significantly from baseline and heart rate increased by 2.3 beats/ min when ExBID was added to basal insulin glargine [27]. The difference was also significant when compared to the placebo. Both ExBID and liraglutide reduce SBP and DBP; however, the increase in heart rate was significantly higher with liraglutide (3.28 vs. 0.69 beats $/ \mathrm{min}$, $P=0.0012$ ) [31].

Similarly, treatment with ExER resulted in a significant reduction in SBP from baseline, ranging from -2.9 to $-4 \mathrm{mmHg}$ in the DURATION 1, 2, 3, 5, and 6 studies, with little or no effect on DBP $[33-35,37,38]$. The improvement in SBP was significantly greater with ExER than with ExBID, sitagliptin, or insulin glargine, but not pioglitazone [33-35, 39]. Mean heart rate was found to be significantly increased from baseline (4 beats/ min, $P<0.05)$ in patients treated with ExER, but not in those treated with glargine [35]. Thus, treatment with either ExBID or ExER consistently results in reduction of SBP.

\section{Effects of Exenatide on Lipid Profile}

The effects of treatment with exenatide on total cholesterol, triglyceride, low-density lipoprotein cholesterol (LDL-C), and high-density lipoprotein cholesterol (HDL-C) have been widely reported in both randomized and observational studies. When ExBID was added to metformin, a SU, or 
the combination, no significant differences in lipid profile were observed in either the ExBID or the placebo group at the end of the initial 30-week period [14-16]. However, further extension of the study to 82 weeks demonstrated a significant increase in HDL-C $(+0.12 \mathrm{mmol} / \mathrm{L})$ and a reduction in triglyceride $(-0.43 \mathrm{mmol} / \mathrm{L})$ from the baseline [17]. The greatest improvement in lipid profile was found in patients with the greatest weight reduction. When patients were followed for up to 3.5 years, a significant improvement was found in all lipid parameters: $5 \%$ reduction in total cholesterol, $6 \%$ in LDL-C, and $12 \%$ in triglyceride, while the HDL-C increased by $24 \%$ [18]. Both total cholesterol and triglyceride concentrations decreased over 26 weeks with both ExBID and glargine, but only LDL-C decreased with ExBID in the HEELA study [23].

In the DURATION studies, treatment with ExER has been shown to improve the lipid profile. Both total cholesterol and LDL-C were significantly reduced with ExER compared to ExBID, while fasting TG was reduced for both treatment modalities [33]. In the DURATION 2 study, HDL-C was significantly improved with ExER, sitagliptin, and pioglitazone, but a reduction in triglyceride was observed only with pioglitazone [34]. The reported findings to date on lipid parameters are variable, and thus further large-scale studies are needed before definite conclusions can be drawn.

\section{SAFETY AND TOLERABILITY}

\section{Gastrointestinal Intolerance}

ExBID is generally well tolerated and the most frequent adverse events were mild-to-moderate GI intolerance, mainly nausea (20-59\%) and vomiting (10-15\%), and were found to be dose dependent and to subside over the first few weeks of initiation [14-21]. Compared to placebo, the occurrence of GI intolerance was higher in the exenatide treatment arm when exenatide was added to metformin, a SU, or a combination of metformin with a SU or TZD. Similarly, GI intolerance was more common with exenatide therapy when exenatide was added to insulin glargine or compared to a combination of glargine and premixed insulin [22-26].

The most common treatment-emergent adverse event reported with ExER treatment was nausea (24-30\%), followed by diarrhea (9-13.5\%), vomiting (4-16\%), constipation (3-10.8\%), and pruritus at the injection site (2-10.8\%) [33-39]. Nausea was reported in significantly fewer patients treated with the once-weekly than the BID preparation [33, 37]. GI intolerance was more common with ExER than with sitagliptin, pioglitazone, or insulin glargine, but less common than with liraglutide $[34,35,38]$.

\section{Hypoglycemia}

In a meta-analysis, mild-to-moderate hypoglycemia was more commonly reported with exenatide than with placebo (16 vs. $7 \%$, relative risk ratio 2.3 ; 95\% CI $1.1-4.9$ ), especially when coadministered with a SU [50]. Eleven percent reported hypoglycemia with combination treatment of exenatide and a TZD [19].

When exenatide was compared to basal insulin glargine or premixed insulin, there was no significant difference in the overall incidence of symptomatic hypoglycemia [22-26]. The incidence of nocturnal hypoglycemia was reported to be lower with exenatide treatment than with insulin [22-25]. The overall hypoglycemia rate was decreased after a reduction of the $\mathrm{SU}$ dose in patients treated 
with the exenatide and insulin combination [25].

Furthermore, addition of exenatide to basal insulin glargine did not increase the risk of hypoglycemia. The number of hypoglycemic events per participant per year did not differ significantly between the exenatide and the placebo group (1.4 vs. $1.2, P=0.49)$ [27]. However, in real-life clinical practice, hypoglycemic events were significantly higher after initiation of exenatide treatment (5.6 vs. $3.3 \%, \quad P<0.001)$ and in insulin-treated compared with non-insulin-treated patients (8.9 vs. $6.1 \%, P<0.001$ ) [28].

It was consistently reported that treatment with ExER did not cause major hypoglycemic episodes [33-39]. The incidence of minor hypoglycemia during both ExER and ExBID treatment was limited to patients using concomitant SU therapy. When ExER was compared to sitagliptin and pioglitazone, the frequency of minor hypoglycemic episodes was low and was similar [34]. Symptomatic hypoglycemia was more common with insulin glargine treatment than with ExER (31 vs. 13\%) [35].

\section{CONCLUSION}

Incretin-based therapies, particularly GLP-1 analogs, offer an alternative option to currently available therapeutic agents in the management of T2DM. Treatment with either ExER or ExBID results in improvement in glycemic control comparable to any other existing agents, without increased risk of major hypoglycemia, and is associated with significant weight loss. It is generally well tolerated, and GI intolerance usually subsides a few weeks after initiation. There is also a trend of improvement in blood pressure and lipid profile, both of which could potentially offer a reduction in cardiovascular events.
Thus, it is a justifiable adjuvant therapy for patients whose disease is not adequately controlled on OADs. Concomitant use with insulin is not licensed yet, although it has proven efficacy in both randomized and real-life clinical practice. To the best of the authors' knowledge, no safety data exist on the use of exenatide in children, pregnant women, or the elderly to inform its safety in such patients, and hence it is not recommended in these patients. However, further continued and robust evaluation, in both randomized and clinical studies, is needed to determine the safety and efficacy of using ExBID and ExER in the long term.

\section{ACKNOWLEDGMENTS}

M. Davies has acted as a consultant, advisory board member, and speaker for Novartis, Novo Nordisk, Sanofi-Aventis, Lilly, Merck Sharp and Dohme, and Roche, and as a speaker for Servier. M. Davies has received grants in support of investigator and investigator-initiated trials from Novartis, Novo Nordisk, Sanofi-Aventis, Lilly, Pfizer, Merck Sharp and Dohme, GlaxoSmithKline, and Servier. K. Khunti has acted as a consultant and speaker for Novartis, Novo Nordisk, Sanofi-Aventis, Lilly, and Merck Sharp \& Dohme. K. Khunti has received grants in support of investigator and investigatorinitiated trials from Novartis, Novo Nordisk, Sanofi-Aventis, Lilly, Pfizer, Boehringer Ingelheim, and Merck Sharp \& Dohme. The authors have no other relevant affiliations or financial involvement with any organization or entity with a financial interest in or financial conflict with the subject matter or materials discussed in the manuscript apart from those disclosed. Dr. Davies is the guarantor for this article, and takes responsibility for the integrity of the work as a whole. 
Conflict of interest. None.

Open Access. This article is distributed under the terms of the Creative Commons Attribution Noncommercial License which permits any noncommercial use, distribution, and reproduction in any medium, provided the original author(s) and source are credited.

\section{REFERENCES}

1. Shaw JE, Sicree RA, Zimmet PZ. Global estimates of the prevalence of diabetes for 2010 and 2030. Diabetes Res Clin Pract. 2010;87:4-14.

2. Gaede P, Lund-Andersen H, Parving HH, et al. Effect of a multifactorial intervention on mortality in type 2 diabetes. N Engl J Med. 2008;358:580-91.

3. NICE. Type 2 Diabetes: The management of type 2 diabetes. http://www.nice.org.uk/nicemedia/pdf/ CG66NICEGuideline.pdf. Accessed 22 Dec 2011.

4. Nathan D, Buse J, Mayer B, et al. Medical management of hyperglycemia in type 2 diabetes: a consensus algorithm for the initiation and adjustment of therapy: a consensus statement of the American Diabetes Association and the European Association for the Study of Diabetes. Diabetes Care. 2009;32:193-203.

5. Nauck M, Vardarli I, Deacon CF, et al. Secretion of glucagon-like peptide-1 (GLP-1) in type 2 diabetes: what is up, what is down? Diabetologia. 2011;54: $10-8$.

6. Bhashyam S, Fields AV, Paterson B, et al. Glucagonlike peptide- 1 increases myocardial glucose uptake via p38alpha MAP kinase-mediated, nitric oxidedependent mechanisms in conscious dogs with dilated cardiomyopathy. Circ Heart Fail. 2010;3: 512-21.

7. Liu X, Pachori AS, Ward CA, et al. Heme oxygenase1 (HO-1) inhibits postmyocardial infarct remodeling and restores ventricular function. FASEB J. 2006;20: 207-16.

8. Anagnostis P, Athyros VG, Adamidou F, et al. Glucagon-like peptide-1-based therapies and cardiovascular disease: looking beyond glycaemic control. Diabetes Obes Metab. 2011;13:302-12.

9. Ban K, Noyan-Ashraf MH, Hoefer J, et al. Cardioprotective and vasodilatory actions of glucagon-like peptide 1 receptor are mediated through both glucagon-like peptide 1 receptordependent and -independent pathways. Circulation. 2008; 117:2340-50.

10. Nyström T, Gonon AT, Sjöholm A, et al. Glucagonlike peptide-1 relaxes rat conduit arteries via an endothelium-independent mechanism. Regul Pept. 2005;125:173-7.

11. Chilton R, Wyatt J, Nandish S, et al. Cardiovascular comorbidities of type 2 diabetes mellitus: defining the potential of glucagon-like peptide-1-based therapies. Am J Med. 2011;124:S35-53.

12. NICE. Liraglutide in the treatment of type 2 diabetes mellitus: clinical utility and patient perspectives. 2011. http://www.nice.org.uk/nicemedia/ live/13248/51259/51259.pdf. Accessed 22 Dec 2011.

13. Eli Lilly. BYDUREON ${ }^{\circledR}$ (exenatide $2 \mathrm{mg}$ powder and solvent for prolonged release suspension for injection) marketing authorisation press release. 2011.http://phx.corporate-ir.net/phoenix.zhtml?c= $101911 \& p=$ irol-newsArticle\&ID=1576430. Accessed 22 Dec 2011.

14. DeFronzo RA, Ratner RE, Han J, et al. Effects of exenatide (exendin-4) on glycemic control and weight over 30 weeks in metformin-treated patients with type 2 diabetes. Diabetes Care. 2005;28:1092-100.

15. Buse JB, Henry RR, Han JJ, et al. Effects of exenatide (exendin-4) on glycemic control over 30 weeks in sulfonylurea-treated patients with type 2 diabetes. Diabetes Care. 2004;27:2628-35.

16. Kendall DM, Riddle MC, Rosenstock J, et al. Effects of exenatide (exendin-4) on glycemic control over 30 weeks in patients with type 2 diabetes treated with metformin and a sulfonylurea. Diabetes Care. 2005;28:1083-91.

17. Blonde L, Klein EJ, Han J, et al. Interim analysis of the effects of exenatide treatment on A1C, weight and cardiovascular risk factors over 82 weeks in 314 overweight patients with type 2 diabetes. Diabetes Obes Metab. 2006;8:436-47.

18. Klonoff DC, Buse JB, Nielsen LL, et al. Exenatide effects on diabetes, obesity, cardiovascular risk factors and hepatic biomarkers in patients with type 2 diabetes treated for at least 3 years. Curr Med Res Opin. 2008;24:275-86.

19. Zinman B, Hoogwerf BJ, Garcia SD, et al. The effect of adding exenatide to a thiazolidinedione in suboptimally controlled type 2 diabetes. Ann Intern Med. 2007;146:477-85.

20. Liutkus J, Rosas GJ, Norwood P, et al. A placebocontrolled trial of exenatide twice-daily added to 
thiazolidinediones alone or in combination with metformin. Diabetes Obes Metab. 2010;12: 1058-65.

21. Pinelli NR, Cha R, Brown MB, et al. Addition of thiazolidinedione or exenatide to oral agents in type 2 diabetes: a meta-analysis. Ann Pharmacother. 2008;42:1541-51.

22. Heine RJ, Van Gaal LF, Johns D, et al. Exenatide versus insulin glargine in patients with suboptimally controlled type 2 diabetes. Ann Intern Med. 2005;143:559-69.

23. Davies MJ, Donnelly R, Barnett AH, et al. Exenatide compared with long-acting insulin to achieve glycaemic control with minimal weight gain in patients with type 2 diabetes: results of the Helping Evaluate Exenatide in patients with diabetes compared with Long-Acting insulin (HEELA) study. Diabetes Obes Metab. 2009;11:1153-62.

24. Bunck M, Diamant M, Corner A, et al. One year treatment with exenatide improves $\beta$-cell function, compared with insulin glargine, in metformin treated type 2 diabetic patients. Diabetes Care. 2009;32:762-8.

25. Barnett AH, Burger J, Johns D, et al. Tolerability and efficacy of exenatide and titrated insulin glargine in adult patients with type 2 diabetes previously uncontrolled with metformin or a sulphonylurea: a multinational, randomized, open-label, twoperiod, crossover noninferiority trial. Clin Ther. 2007;29:2333-48.

26. Nauck M, Duran S, Kim D, et al. A comparison of twice-daily exenatide and biphasic insulin aspart in patients with type 2 diabetes who were suboptimally controlled with sulfonylurea and metformin: a non-inferiority study. Diabetologia. 2007;50:259-67.

27. Buse JB, Bergenstel RM, Glass LC, et al. Use of twice daily exenatide in basal insulin-treated patients with type 2 diabetes. A randomised controlled trial. Ann Intern Med. 2011;154:1-10.

28. Thong KY, Jose B, Sukumar N, et al. Safety, efficacy and tolerability of exenatide in combination with insulin in the Association of British Clinical Diabetologists nationwide exenatide audit. Diabetes Obes Metab. 2011;13:703-10.

29. Yoon NM, Cavaghan MK, Brunelle RL, et al. Exenatide added to insulin therapy: a retrospective review of clinical practice over 2 years in an academic endocrinology outpatient setting. Clin Ther. 2009;31:1511-23.

30. Sheffield CA, Kane MP, Busch RS, et al. Safety and efficacy of exenatide in combination with insulin in patients with type 2 diabetes mellitus. Endocr Pract. $2008 ; 14: 285-92$.

31. Buse JB, Rosenstock J, Sesti G, et al. Liraglutide once a day versus exenatide twice a day for type 2 diabetes: a 26-week randomised, parallel-group, multinational, open-label trial (LEAD-6). Lancet. 2009;274:39-47.

32. Larsen J, Hylleberg B, $\mathrm{Ng} \mathrm{K}$, et al. Glucagon-like peptide- 1 infusion must be maintained for $24 \mathrm{~h} /$ day to obtain acceptable glycemia in type 2 diabetic patients who are poorly controlled on sulphonylurea treatment. Diabetes Care. 2001;24:1416-21.

33. Drucker DJ, Buse JB, Taylor K, et al. Exenatide once weekly versus twice daily for the treatment of type 2 diabetes: a randomised, open-label, non-inferiority study. Lancet. 2008;372:1240-50.

34. Bergenstal RM, Wysham C, MacConell L, et al. Efficacy and safety of exenatide once weekly versus sitagliptin or pioglitazone as an adjunct to metformin for treatment of type 2 diabetes (DURATION-2): a randomised trial. Lancet. 2010;376:431-9.

35. Diamant M, Goal LV, Stranks S, et al. Once weekly exenatide compared with insulin glargine titrated to target in patients with type 2 diabetes (DURATION-3): an open-label randomised trial. Lancet. 2010;375:2234-43.

36. Russell-Jones D, Cuddihy RM, Hanefeld M, et al. Efficacy and safety of exenatide once weekly versus metformin, pioglitazone, and sitagliptin used as a monotherapy in drug-naïve patients with type 2 diabetes (DURATION-4): a 26-week double-blind study. Diabetes Care. 2012;35:252-8.

37. Blevins T, Pullman J, Malloy J, et al. DURATION-5: exenatide once weekly resulted in greater improvements in glycemic control compared with exenatide twice daily in patients with type 2 diabetes. J Clin Endocrinol Metab. 2011;96:1301-10.

38. Buse J, Nauck M, Frost T, et al. Efficacy and safety of exenatide once weekly versus liraglutide in subjects with type 2 diabetes (DURATION 6): a randomised, open-label study. Diabetologia. 2011;54:S38.

39. Buse J, Drucker DJ, Taylor KL, et al. DURATION-1: Exenatide once weekly produces sustained glycemic control and weight loss over 52 weeks. Diabetes Care. 2010;33:1255-61.

40. Holman RR, Paul SK, Bethel MA, et al. 10-Year follow-up of intensive glucose control in type 2 diabetes. N Engl J Med. 2008;359:1577-89.

41. The ACCORD study group, Gerstein HC, Miller ME, et al. Action to Control Cardiovascular Risk in Diabetes Study Group (ACCORD). Effects of 
intensive glucose lowering in type 2 diabetes. N Engl J Med. 2008;358:2545-59.

42. The ADVANCE collaborative group, Patel A, MacMahon $S$, et al. Intensive blood glucose control and vascular outcomes in patients with type 2 diabetes. N Engl J Med. 2008;48:2560-72.

43. Bonora E, Muggeo M. Postprandial blood glucose as a risk factor for cardiovascular disease in type II diabetes: the epidemiological evidence. Diabetologia. 2001;44:2107-14.

44. Williamson DF, Thompson TJ, Thun M, et al. Intentional weight loss and mortality among overweight individuals with diabetes. Diabetes Care. 2000;23:1499-504.

45. Best JH, Hoogwerf BJ, Herman WH, et al. Risk of cardiovascular disease events in patients with type 2 diabetes prescribed the GLP-1 receptor agonist exenatide twice daily or other glucose-lowering therapies: a retrospective analysis of the LifeLink $^{\text {TM }}$ database. Diabetes Care. 2010;34:90-5.
46. Peskin B, Shcheprov A, Boye K, et al. Cardiovascular outcomes associated with a new once-weekly GLP-q receptor agonist vs. traditional therapies for type 2 diabetes: a simulation analysis. Diabetes Obes Metab. 2011;13:921-7.

47. ClinicalTrials.gov. EXenatide Study of Cardiovascular Event Lowering (EXSCEL). http://clinicaltrials.gov/ ct2/show/NCT01144338. Accessed 22 Dec 2011.

48. Hirata K, Kume S, Araki S, et al. Exendin-4 has an anti-hypertensive effect in salt-sensitive mice model. Biochem Biophys Res Commun. 2009;380: $44-9$.

49. Okerson T, Yan P, Stonehouse A, et al. Effects of exenatide on systolic blood pressure in subjects with type 2 diabetes. Am J Hypertens. 2010;23: 334-9.

50. Amori RE, Lau J, Pittas AG. Efficacy and safety of incretin therapy in type 2 diabetes: systematic review and meta-analysis. JAMA. 2007;298:194-206. 